\title{
Tumorendoprothetik am distalen Femur: Indikationen und operative Technik
}

\author{
Hagen Fritzsche, Christine Hofbauer, Klaus-Dieter Schaser
}

\section{Zusammenfassung}

Das distale Femur ist neben dem proximalen Femur und der proximalen Tibia ein Prädilektionsort für die Manifestaion von primär malignen und benignen/aggressiven Knochentumoren [11] der Extremitäten sowie eine Hauptlokalisation für metastatische Destruktionen. Bei malignen Skeletttumoren des distalen Femurs ist eine rasche Einleitung der Diagnostik zur Abklärung der Tumorbiologie (Entität und Grading) von essenzieller Bedeutung und determiniert die weitere onkologische Systembehandlung und tumororthopädische Lokaltherapie. Fehlerhaft durchgeführte Biopsien können falsch-negative Diagnosen erbringen und führen - wenn nicht nach bestimmten Richtlinien durchgeführt zu schlechtem funktionellem Ergebnis nach Resektion, können den Extremitätenerhalt gefährden und beeinträchtigen die Prognose des Patienten. Chirurgisch stehen nach intra-/extraartikulärer weiter Resektion heutzutage vielfältige Rekonstruktionsmöglichkeiten zur Verfügung. Der tumorendoprothetische Ersatz stellt dabei das am häufigsten durchgeführte Rekonstruktionsverfahen nach Exzision kniegelenknaher Tumoren des distalen Femurs dar. Durch den distalen Femurersatz ist ein Extremitätenerhalt mit funktioneller und belastungsstabiler Wiederherstellung der Kniegelenkbeweglichkeit möglich. Er findet Anwendung zur Rekonstruktion bei massiven gelenknahen osteolytischen Destruktionen, bei pathologischen Frakturen, periprothetischen Frakturen oder in der Revisionsendoprothetik. Den Vorteilen einer raschen Mobilisation, sofortigen Vollbelastung, guter Funktion und kurzen Rehabilitationszeit stehen die zu erwartenden Revisionseingriffe bei erhöhter Komplikationsrate mit erhöhtem Infektionsrisiko (Megaprothese) sowie aseptische Lockerungen und Implantatversagen (inkl. Kopplungsmechanismus und PE-Inlays) gegenüber.

\section{Tumour Endoprosthesis on the Distal Femur: Indications and Surgical Technique}

Along with the proximal femur and the proximal tibia, the distal femur is the most affected site for the manifestation of primary malignant and benign/aggressive bone tumours [11] of the extremities, as well as being a principle site of metastatic destruction. In malignant, skeletal tumors of the distal femur, rapid initiation of complete diag- nostic measures is essential for assessment of tumour biology (entity and grading) and guidance of the further oncological treatment, including both systemic and local orthopaedic therapy. Incorrectly performed biopsies can lead to false negative diagnosis, usually result in poor functional outcome, can compromise salvage of the extremity and adversely affect the patient's prognosis. After intra- or extraarticular resection, a wide range of surgical options is available. Following excision of tumours around the knee joint, endoprosthetic distal femur replacement is the most frequently performed reconstruction procedure and allows functional restoration of knee joint mobility. Distal femur replacement is typically performed in patients with massive osteolytic destruction, in pathological and periprosthetic fractures or for revision of total knee replacements. Rapid mobilisation without loading restrictions, a good range of motion and short rehabilitation periods are the advantages of the procedure. However, in comparison to conventional knee arthroplasty, distal femur replacement is associated with increased rates of complication, such as infection, aseptic loosening and hardware failure.

\section{Einleitung}

Primäre Knochen- und Weichteiltumoren sowie tumorähnliche Läsionen sind seltene Erkrankungen. Insbesondere bei malignen Entitäten ist das Zeitintervall zwischen Beginn der klinischen Symptomatik, nach bestimmten Richtlinien durchgeführter Biopsie, adäquat kom-

OP-JOURNAL 2016; 32: 221-230

(c) Georg Thieme Verlag KG Stuttgart · New York DOI http://dx.doi.org/10.1055/s-0036-1595900 plettierter Umfelddiagnostik und Einleitung der spezifischen systemischen und lokalen Therapiemaßnahmen entscheidend für die Langzeitprognose.

Bei frühzeitiger Diagnose und optimalem Management sind bei primär malignen Knochentumoren der Extremitäten auch infolge verbesserter Resektionstechniken und optimierter neoadjuvanter und adjuvanter Systemtherapien in bis zu 90\% extremitäterhaltende Versorgungen in kurativer Intention möglich [16]. Bei verzögertem Management, falsch durchgeführter Biopsie, intraläsionaler Resektion ist ein hohes Lokalrezidivrisiko zu befürchten, ein unbefriedigendes funktionelles Ergebnis zu erwarten oder aber auch in seltenen Fällen die Amputation nicht zu vermeiden. Aufgrund der Seltenheit der Erkrankungen (Bsp.: Inzidenz des Osteosarkoms 150 Neuerkrankungen/Jahr in Deutschland, davon über die Hälfte am distalen $\mathrm{Fe}$ mur) und der hochspeziellen Resektionstechniken und Verfahren in der Defektrekonstruktion bestehen nur an muskuloskeletalen Zentren mit tumor- 
orthopädischer/-chirurgischer Expertise hierzu entsprechende Erfahrungen. Die Behandlung muss in solchen Zentren im interdisziplinären Ansatz unter Einbindung aller am multimodalen Therapiekonzept beteiligten Fachdisziplinen (internistische Onkologie, Radioonkologie, Pathologie etc.) geplant und durchgeführt werden.

Primäre Knochentumoren und Skelettmetastasen manifestieren sich an den langen Röhrenknochen am häufigsten am Femur [11]. In unterschiedlichem Ausmaß befallen diese Tumoren dabei den Abschnitt des proximalen, meta-/ diaphysären oder distalen Femurs. Prädilektionsort der Osteosarkome ist in der Mehrzahl der Fälle die distale Metaphyse (75\%), während Ewing-Sarkome und Chondrosarkome vermehrt im proximalen Femur beobachtet werden [11]. Pathologische Frakturen infolge sekundärer Knochenmetastasen sind mit $>50 \%$ am Femur sehr häufig [18].

In einem interdisziplinären Behandlungskonzept wird die Therapie mit der bestmöglichen Prognose für den Patienten geplant. Hierbei beeinflusst eine Vielzahl von Faktoren die individuelle Therapieplanung. Tumorabhängige Faktoren sind neben Tumorbiologie (Dignität, Grading, Response auf Chemotherapie), Tumorlokalisation (intra-/vs. extraartikulär), Tumorgröße (Weichteilbeteiligung/paraossales Tumorsubstrat), Beteiligung neurovaskulärer Strukturen auch das Ausbreitungsstadium (solitär vs. primär metastasiert).

Patientenspezifische Determinanten sind neben Alter und funktionellem Anspruch auch das Vorliegen von zusätzlichen Erkrankungen. Die Planung des onkologischen Konzepts erfolgt über ein interdisziplinäres Tumorboard anhand der o.g. Faktoren, welches die Indikation und das Timing der Durchführung (neo)adjuvanter Therapieverfahren (Chemotherapie, Strahlentherapie, Hyperthermie, Extremitätenperfusion) verifiziert und die Art/Radikalität der Lokaltherapie festlegt. Das onkochirurgische Spektrum reicht von palliativen Stabilisierungen, intraläsionalen Verfahren bei benignen Tumoren oder tumorähnlichen Läsionen mit konservativem Regime (leave me alone lesion), über Verbundosteosynthesen bis hin zu radikalen weiten intraund extraartikulären Resektionen unter Einschluss meta-diaphysärer Segmente (lokal aggressive oder Low-/High-GradeTumoren). Klassische Kompartimentre- sektionen (was bei Tumormanifestationen im Bereich des distalen Femurs eine Entfernung des gesamten Knochens bedeuten würde) haben bez. Prognose und Lokalrezidivrate keinen Vorteil gezeigt [9], zeigen aber funktionell massiv schlechtere Ergebnisse.

Die anschließende Rekonstruktion des ossären Defekts des distalen Femurs erfolgt bei der Möglichkeit zum Gelenkerhalt entweder biologisch (autologer freier oder vaskularisierter Knochentransfer, mittels Verbundosteosynthesen oder durch Segmenttransport bzw. Distraktionsosteogenese) oder aber bei Gelenkbeteiligung tumorendoprothetisch (distaler/totaler Femurersatz).

Bei palliativen Intentionen (disseminiertes Sarkom, multiple Karzinommetastasen, Plasmozytom etc.) mit drohender/manifester pathologischer Fraktur werden nach ggf. intraläsionalen Exzisionen reguläre Osteosynthesetechniken (Marknägel, winkelstabile Platten) ohne/ mit Verbundosteosynthese oder auch tumorendoprothetische Therapieverfahren eingesetzt.

Bei ausgedehnten und seltenen Befunden mit Invasion des Großteils der kniegelenknahen Muskulatur, des Streckapparats, der Gefäße, aber ohne Beteiligung der neuralen Strukturen kann eine Segmentresektion in Form einer Umkehrplastik erwogen werden. Bei onkologisch suffizienter Resektion sind die funktionellen Defizite vergleichbar zu denen nach proximaler Unterschenkelamputation und deutlich geringer als nach proximaler Oberschenkelablation oder Hüftgelenkexartikulation.

Als Ultima Ratio bleiben bei onkologisch nicht sicher resektablen Läsionen, Infiltration neurovaskulärer Strukturen und/ oder fehlender realistischer Aussicht auf onkologisch suffiziente Resektion (mehrfache, intraläsionale Eingriffe in der Anamnese, wiederholte Lokalrezidive bei schlechter Response auf systemische Therapieverfahren, absehbar funktionslose Extremität, nicht anderweitig beherrschbare Schmerzzustände etc.) die ablativen Verfahren bestehen. Am distalen Femur ist dies heutzutage nur noch in $5-15 \%$ der Fälle notwendig $[19,20]$.

\section{Diagnostik}

Osteogene Sarkome oder Knochenmetastasen können im Allgemeinen über ein längeres Intervall asymptoma- tisch verlaufen. In Abhängigkeit von der Lokalisation, der Ausdehnung eines paraossären Weichteilanteils und/oder der Beteiligung neurovaskulärer Strukturen werden sie unterschiedlich früh oder spät klinisch auffällig. Im Bereich des distalen Femurs führen unklare Schwellungen mit oder ohne assoziierte Kniegelenkbeschwerden häufig zum ersten Arztkontakt und zur primären Diagnostik. Die Patienten klagen meist vorwiegend über dumpfe, belastungsabhängige Beschwerden. Oft ist der Nachtschmerz klinisch vordergründig. Bagatelltraumata und sportliche Aktivitäten werden häufig als Erklärung der Beschwerden akzeptiert und verzögern genauso wie fehlinterpretierte Irritationen neurovaskulärer Strukturen die frühzeitige Diagnose. Pathologische Frakturen sind am distalen Femur nicht selten das Erstsymptom eines primär malignen Knochentumors oder einer Knochenmetastase [7]. Systemische Beschwerden (Fieber, Schüttelfrost, Gewichtsverlust, Abgeschlagenheit und Appetitlosigkeit) treten eher selten und wenn, dann zumeist erst verzögert auf.

Radiologisch soll immer zuerst eine konventionelle Röntgenaufnahme des Femurs in 2 Ebenen erfolgen.

Nicht selten wird aufgrund unklarer Kniegelenkbeschwerden auch eine MRT-Untersuchung initial durchgeführt. Sowohl das angrenzende Knie- als auch Hüftgelenk sollen röntgenologisch möglichst mit abgeklärt werden. Durch die Reaktion des Knochens auf bestimmte aggressive Tumoren, aber auch durch die individuelle Tumormatrix (Osteoidbildung) ergeben sich im Röntgenbild oft typische, pathognomonische, radiomorphologische Erscheinungsbilder, die Rückschlüsse auf Entität und Dignität erlauben. In Zusammenschau mit Alter und klinischer Symptomatik erfolgt anschließend die gezielte Indikationsstellung über Notwendigkeit, Art und Zügigkeit eines anschließenden Schnittbildverfahrens. Die CT ermöglicht eine optimale Darstellung der Ausdehnung des Knochenprozesses, aber auch der Tumormatrix (fibrös, kalzifizierend etc.) und wird zur Stabilitätsbeurteilung herangezogen. In Kombination mit der Kontrastmittelanwendung (KM bei Angio-CT mit multiplanaren Rekonstruktionen) wird zudem die Gefäßbeteiligung beurteilbar. 
Goldstandard bei der erweiterten Diagnostik primär maligner Knochentumoren, vor allem zur Beurteilung der Markraumausdehnung und Weichteilbeteiligung, bleibt die MRT des gesamten Kompartiments.

Der Vorteil liegt in der hohen Weichteilkontrastierung und damit besseren Beurteilbarkeit sowohl der intra- als auch extraossären Tumorausdehnung. Die eventuelle Beteiligung neurovaskulärer Strukturen (Angio-MRT) wird, wie auch mögliche Skip-Metastasen (Läsionen ohne Verbindung zum Primärtumor, aber im selben Kompartiment), erfasst. Weiterhin ist die MRT essenziell zur Planung einer Biopsie, da heterogene Binnenmuster eines Tumors ebenso wie das unterschiedliche KM-Aufnahmeverhalten sowie Nekroseareale, liquide Formationen etc. dargestellt werden, die hinweisend auf ein höheres Grading sind. Zudem erlauben bestimmte fettgesättigte Sequenzen Rückschlüsse auf den Fettgehalt des Tumorgewebes, andere Sequenzen wiederum sind hinweisend für chondroide Gewebeanteile. Wegen der o.g. Fakten sollte die MRT immer als Kompartiment-MRT primär und nach neoadjuvanter Therapie durchgeführt werden. Über die Änderung des Binnenmusters und der Regression/Progression der Tumorgröße können nicht selten auch Informationen über die Response auf die neoadjuvante Polychemotherapie erhalten werden.

Die Skelettszintigrafie dient der Erfassung multipler skeletaler Läsionen wie Skelettmetastasen oder multifokale Manifestationen primärer Knochentumoren. Die Beurteilung einer Läsion kann bei epi-/metaphysärer Lage im Kindesalter aufgrund der physiologischen Anreicherung der Wachstumsfuge schwierig sein.

Im Verbund mit einer Positronenemissionstomografie (PET) bzw. ${ }^{18}$ Fluor-Desoxyglukose-PET (FDG-PET) kann die CT-/MRT-Untersuchung eine optimierte Umfelddiagnostik bieten und die metabolische Aktivität intramedullärer/paraossaler Tumorausdehnungen/fraglicher Skip-Metastasen vor und nach Chemotherapie beurteilen.

\section{Biopsie}

Die Indikation zur Biopsie ergibt sich bei klarem Verdacht auf maligne-/benigneaggressive Läsionen. Typische radiomorphologisch eindeutig zuordenbare Läsio- nen (sog. leave me alone lesions) benötigen keine bioptische Sicherung. Bleibt die Dignität unklar, muss ein Zweittumor ausgeschlossen werden; sind Gewebeproben erforderlich, um den Therapieplan anzupassen (Bsp. Rezeptorstatus bei Mammakarzinom), muss ebenfalls zwingend eine histologische Sicherung erfolgen.

Das Timing der Biopsie besteht erst nach Abschluss aller bildgebenden Diagnostik, da inzisionsbioptisch bedingte intratumorale Hämatome Nekrosen und damit ein höheres Grading vortäuschen können.

Die grundsätzlichen Prinzipien zur Durchführung einer Biopsie sind unabhängig von der Lokalisation und werden daher auch am Femur konsistent nach den folgenden onkologischen Grundregeln durchgeführt:

- Zugang in Schnittführung der Definitiv-OP

- Zugang in Längsrichtung der Extremitäten

- direkter Zugang (kürzester Weg) zum Tumor unter Berücksichtigung der später notwendigen En-bloc-Entfernung des Biopsiekanals mit Tumorresektat

- keine Eröffnung zusätzlicher muskulärer/artikulärer Kompartimente

- keine Biopsie durch Muskelgruppen, die später zur Weichteilrekonstruktion (Lappendeckung) benötigt werden

- keine Kontamination von Gefäß-/Nervenstrukturen

- repräsentative und mengenmäßig ausreichende Gewebsprobe (vitales Tumorgewebe)

Die offene Biopsie bleibt der Goldstandard, da danach eine sichere Diagnose mit einer diagnostischen Rate von ca. 98\% erwartet werden kann [36]. Intraoperative Schnellschnittuntersuchungen erhöhen die diagnostische Sicherheit und sind als Standard anzusehen, da falsch-negative Proben (Tumornekrosen, Hämatome, ödematöses Gewebe aus der reaktiven Randzone etc.) vermieden werden können.

Daher sollte eine Biopsie bei V.a. primär maligne und/oder lokal-aggressive benigne Tumoren zwingend an einem Zentrum für Tumororthopädie/-chirurgie durchgeführt werden.

Nach einer Studie der Musculoskeletal Tumor Society liegt die Fehlerquote bei onkochirurgischen Eingriffen außerhalb solcher Zentren bei bis zu 30\%, während in Einrichtungen mit entsprechender Expertise diese nur bei $9 \%$ liegt [32]. Nach Biopsie und Klärung des onkologischen Konzepts erfolgt ggf. die onkochirurgische Resektion. Infolge der Kontamination mit Tumorzellen muss der Biopsiekanal inklusive Biopsienarbe am Resektat verbleibend en bloc mitreseziert werden.

Eine nicht korrekt durchgeführte Biopsie verzögert oder verfälscht die Diagnostik, beeinflusst das anstehende Resektionsverfahren mit ggf. unnötigerweise erforderlicher Erweiterung der Resektion und konsekutiv vergrößerten Resektionsdefekten mit schlechterem funktionellem Ergebnis. Im ungünstigsten Fall ist der Erhalt der Extremität gefährdet und die Gesamtprognose des Patienten wird negativ beeinflusst.

Bei Knochentumoren am distalen Femur wird die Biopsie überwiegend von lateral oder alternativ von anteromedial durchgeführt. Anteriore Zugänge durch den Streckapparat müssen genauso wie dorsale Biopsiezugänge in Gefäß-/Nervennähe möglichst unter den o.g. Bedingungen vermieden werden.

\section{Resektion}

Die Behandlung bei Tumoren am distalen Femur ist vielfältig und unterscheidet sich vordergründig von der onkologischen Intention. Das Ausmaß der Resektion ist dabei abhängig von folgenden tumorassoziierten Faktoren:

- vorhandene Tumorbiologie (Dignität, histopathologische Entität, Grading)

- anatomische Lokalisation/Wachstumsmuster des Tumors (extra-/intrakompartimental, intra-/extraartikulär)

- topografische Lagebeziehung des Tumors zu neurovaskulären bzw. muskulären Strukturen

- Stadium der zugrunde liegenden Tumorerkrankung (solitäre Läsion vs. metastasierte)

- Response des Tumors auf neoadjuvante Therapien

Die Planung der Operation erfolgt anhand des erforderlichen angestrebten Resektionsrands (intraläsional, marginal, weit, radikal). 


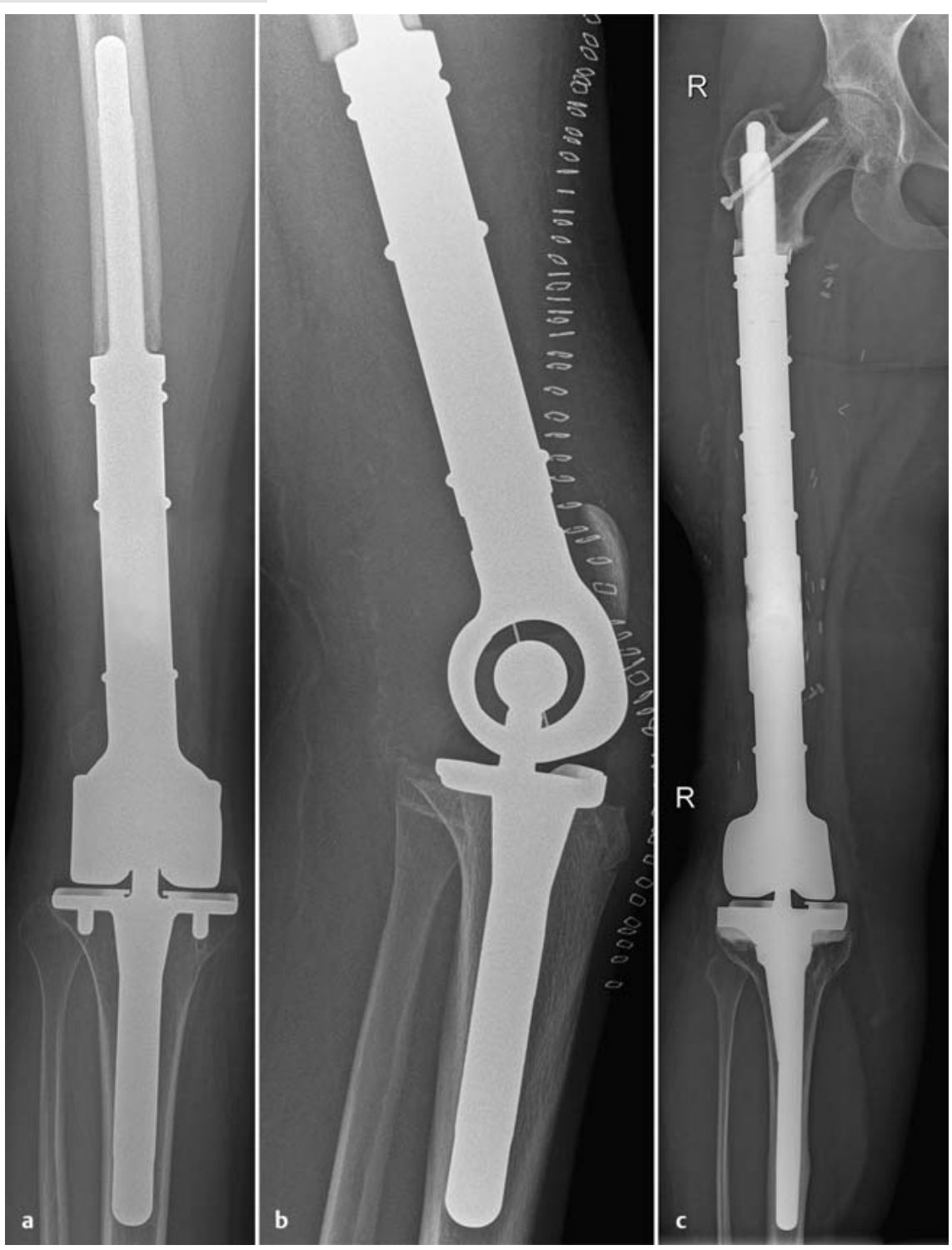

Abb. $\mathbf{1}$ a bis c a, b distaler Femurersatz bei einem 16-jährigen Patienten nach Resektion eines Osteosarkoms. c Sonderanfertigung eines proximal verriegelbaren distalen Femurersatzes aufgrund längerer Rekonstruktionsstrecke bei ausgedehnter Tumorgröße (Ewing-Sarkom) bei einer 20-jährigen Patientin.

\section{Sarkome (Low-/High-Grade-Tumoren) und solitäre Metastasen biologisch günstiger Tumorentitäten (Bsp. Nierenzellkarzinom)}

Hier müssen weite, lokale oder sogar extrakompartimentelle Resektionen durchgeführt werden. Zeigt der Tumor bildmorphologisch keine Infiltration des Kniegelenks, kann die Resektion intraartikulär durchgeführt werden (Abb.1). Andernfalls muss eine extraartikuläre und somit extrakapsuläre Resektion unter Mitnahme des gesamten Kniegelenks ohne Eröffnung desselben (inkl. des gesamten Bandapparats, transpatellare Osteotomie, koronare Teilresektion der
Quadrizepssehne etc.) erfolgen (Abb. 2). Allerdings sind direkte Invasionen von Tumoren in das Kniegelenk selten.

Ein maligner Erguss, intraartikuläres Tumorgewebe und zuvor durchgeführte intraartikuläre Operationen sind, neben fehlerhaft durchgeführten Biopsien mit primärer Kontamination des Gelenks sowie pathologischen Frakturen, Indizien und Gründe für eine extraartikuläre Resektion.

Ist kein sinnvolles funktionelles Ergebnis zu erwarten, muss eine Arthrodese, Segmentresektion (Umkehrplastik) oder gar Ablatio erwogen werden [13].

\section{Aggressive/benigne Läsionen}

Die meisten benignen Läsionen und tumorähnlichen Läsionen am Femur sind Zufallsbefunde und bedürfen nicht in jedem Fall einer operativen Therapie. Viele der typischerweise am Femur auftretenden gutartigen Befunde sind im radiologischen Erscheinungsbild oftmals eindeutig und kommen nach Abschluss des Skelettwachstums komplett zum Stillstand (z.B. nicht ossifizierendes Knochenfibrom). Eine radiologische Kontrolle oder histologische Sicherung ist oft nicht notwendig („leave me alone lesions“) [37]. Sollte in seltenen Fällen durch ausgedehnte Befunde eine Stabilitätsgefährdung oder aber pathologische Fraktur eintreten, ist dann entweder eine protektive Stabilisierung oder aber eine Rekonstruktion mit entsprechender Osteosynthese indiziert.

Aktive und lokal aggressive gutartige Läsionen (z. B. Riesenzelltumoren, aneurysmatische Knochenzysten, aggressive Osteoblastome etc.) erfordern aufgrund der erhöhten Rezidivrate nach intraläsionaler Resektion entweder eine marginale Resektion (in seltenen Fällen auch eine weite En-bloc-Exzision) oder aber die Kombination aus intraläsionaler Resektion mit einem Adjuvans. Am häufigsten kommt hierbei die lokale Zementauffüllung zur Anwendung [37]. Nur in äußerst seltenen Fällen (ausgedehnte lokale Läsionen, nicht rekonstruierbare pathologische Frakturen mit Defekten oder wiederholte Rezidive) kann ein distaler Femurersatz bei benignen Läsionen indiziert sein.

\section{Palliative Indikationen/Metastasen/ pathologische Frakturen}

Indikationen für den distalen Femurersatz sind neben großen metastatischen Destruktionen auch pathologische Frakturen und ausgedehnte ossäre Defekte, die sich osteosynthetisch mit oder ohne Zement nicht mehr übungsstabil rekonstruieren lassen.

In der Palliativsituation mit Instabilität des distalen Femurs werden bei intakter Gelenkfläche und ausreichender kondylärer/interkondylärer metaphysärer Knochensubstanz primär Kürettagen mit anschließender Zementauffüllung mit oder ohne zusätzliche vorzugsweise winkelstabile Plattenosteosynthesen angewendet. Ein osteolytischer Aufbrauch der gesamten distalen Femurepi-/-metaphyse oder Beteiligung großer Gelenkflächen- 

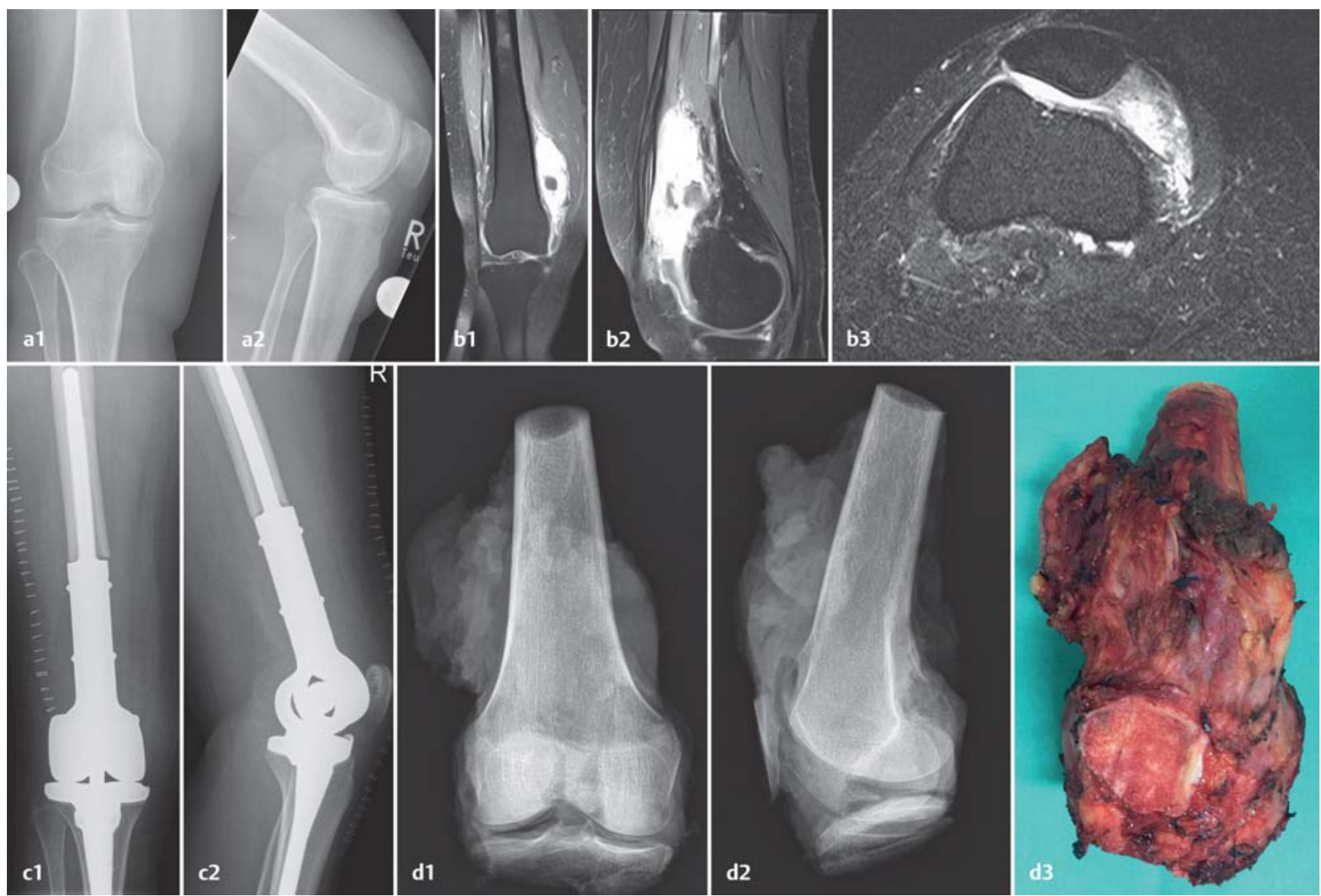

Abb. 2 a bis d 64-jährige Patientin: undifferenziertes pleomorphes Weichteilsarkom mit Kniegelenkinfiltration des Recessus suprapatellaris rechts und malignem Erguss. Initial neoadjuvante CTx, dann schlechte Response und Planung zur extraartikulären Resektion mit nachfolgendem distalem Femurersatz. a konventionelles Röntgen, b MRT, c postoperative Röntgenkontrolle und $\mathbf{d}$ Röntgenbildgebung und klinisches Makrofoto des Resektats.

anteile ohne suffiziente Möglichkeit einer Schraubenverankerung indiziert zum Erhalt der Mobilität den distalen Femurersatz. Bei radikaler Tumorresektion sind gute klinische Ergebnisse mit Reduktion der Komplikationsrate zu erwarten [18].

\section{Rekonstruktion}

Infolge der verbesserten (neo)adjuvanten und operativen Therapiemaßnahmen hat sich die Gesamtüberlebensrate bei primär malignen Knochentumoren deutlich gebessert (61-92\%) [16], wodurch die Langzeitergebnisse der endoprothetischen Rekonstruktionen und deren spezifische Komplikationen zunehmend an Relevanz gewinnen.

Bei der Wahl der Rekonstruktion gibt es eine Vielzahl an operativen Verfahren, die eine individualisierte Patientenversorgung erlauben. Verschiedene Formen der modularen Tumorendoprothetik stehen diversen Formen der biologischen
Rekonstruktion gegenüber. Komplette biologische Verfahren sind Autografts (gefäßgestielte Fibula), Allografts oder deren Kombination. Den Vorteilen biologischer Systeme mit hoher Regenerationsfähigkeit, Belastbarkeitsadaptation, partieller Wachstumsfugenausgleich stehen die verlängerte Einheilungszeit, längerfristige Ent- bzw. Teilbelastung und Notwendigkeit externer Stabilisatoren (Orthesen) gegenüber. Eine Sonderform stellen Compositeallografts dar, bei denen ein Allograft mit einer Endoprothese kombiniert wird. Alleinige osteoartikuläre Allografts sind aufgrund hoher Komplikations- und Versagerraten obsolet geworden. Eine zunehmende Renaissance findet vereinzelt das Verfahren der extrakorporalen Bestrahlung des tumorbefallenen Knochens mit anschließender Replantation im Sinne eines Autografts [39]. Insgesamt gilt es, die individuellen Gegebenheiten des Patienten und v.a. in kurativer Intention das Erreichen eines onkologisch weiten Resektionsrands zu beachten. Daran orientierend erfolgt die
Planung der Rekonstruktion, die Beachtung der Faktoren Rekonstruktionslänge, Gelenksituation, zu erwartende Belastungssituation im Kontext zu Begleiterkrankungen, Notwendigkeit und Art einer adjuvanten Therapie, Prognose, Alter (Wachstumsfugen) sowie Weichteilsituation.

Tumorendoprothetische Verfahren erlauben die Rekonstruktion epi-/meta-/ diaphysärer Knochendefekte mit der Möglichkeit eines vollständigen Gelenkersatzes. Infolge der Verfügbarkeit modularer Implantate kann intraoperativ jede Situation individuell modifiziert werden. Vorteil ist eine sofortige stabile Situation, die eine kurzfristige Vollbelastbarkeit erlaubt. Die im Baukastensystem zur Verfügung stehenden modularen Systeme gewährleisten eine stufenweise Adaptation an aktuelle Rekonstruktionslänge und Rotation und steigern somit die intraoperative Flexibilität [40]. Durch bessere onkologische Therapieoptionen mit verbesserten Überlebenszeiten der 
Patienten werden an die Tumorendoprothesen hohe Ansprüche bez. Standzeiten, Implantatversagen, Lockerungsverhalten, Infektionsrisko und funktionellem $\mathrm{Er}$ gebnis gestellt.

Die Implantatverankerung erfolgt nahezu immer intramedullär. Spezielle Oberflächenstruktureigenschaften und Beschichtungen erhöhen die Osteointegration und resp. die Langzeitstabilität und reduzieren die Infektanfälligkeit [22]. Zementfreie Verankerungen werden vor dem Hintergrund einer ggf. notwendigen Wechseloperation bevorzugt. Bei geplanter postoperativer Bestrahlung oder fortgeschrittenem Patientenalter ist die zementierte Variante zu empfehlen [39].

\section{Distaler Femurersatz}

Grundvoraussetzungen für eine endoprothetische Rekonstruktion mit distalem Femurersatz nach Tumorresektion sind die Möglichkeit der suffizienten Verankerung des Prothesenschafts in der Meta-/Diaphyse sowie eine adäquate Weichteildeckung und Rekonstruktion einer funktionsfähigen, kniegelenkumgreifenden Streck- und Beugemuskulatur.

Die Resektionsnotwendigkeit primärer und sekundärer muskuloligamentärer Stabilisatoren (Kollateral-/Kreuzbänder, Lig. patellae, M. quadriceps) führt $\mathrm{zu}$ einer Instabilität mit gesteigertem biomechanischem Stress für den endoprothetischen Ersatz. Für die Rekonstruktion wird ein kinematisches Rotationsgelenk benötigt, welches neben einer suffizienten Valgus-/Varusstabilität eine gute Flexion/Extension sowie minimale Rotation erlaubt $[13,14,26,30,40]$. Bewährt haben sich dabei unterschiedliche Systeme. Eines der häufigsten für diese Indikation verwendeten Systeme ist das MUTARS ${ }^{\circledR}$-System (Implantcast ${ }^{\circledR}$, Buxtehude, Deutschland), das diese Anforderungen und Eigenschaften inkludiert und zudem durch die Möglichkeit der Silberbeschichtung eine verbesserte Infektprophylaxe bietet $[8,14,22,24]$. Während in der Primärendoprothetik die 10Jahres-Standzeiten für Knie- und HüftTEP um 90\% liegen, zeigen die distalen Femurrekonstruktionen ein mittelfristiges Überleben von < 70\% [34]. Eine resultierende Funktion mit ca. 80\% der physiologischen Norm ergibt eine akzeptable Patientenzufriedenheit $[14,26,30]$. Trotz einer im Vergleich zur Primärendoprothetik deutlich höheren Komplikationsrate ist langfristig der Erhalt der
Extremität mit suffizienter Funktion möglich, wenngleich in der Folge Revisionseingriffe zu erwarten sind $[4,16]$.

Tumorendoprothesen werden auch bei nicht onkologischen Patienten verwendet. Bei höhergradigen, knöchernen und gelenknahen Defektsituationen distaler Femurfrakturen oder nach vorangegangener gescheiterter endoprothetischer oder osteosynthetischer Versorgung bietet der distale Femurersatz eine suffiziente Versorgungsmöglichkeit mit modularer Adaptation an die jeweilige individuelle Defektkonstellation des Patienten [38]. Bei akzeptablen klinischen Ergebnissen ähnelt die Komplikationsrate denen nach onkologischen Rekonstruktionen [28]. Durch eine i.d. R. sofortige Vollbelastbarkeit können insbesondere beim älteren Patienten längerfristige Immobilisationen und somit sekundäre Komplikationen vermindert werden. Ähnlich wie bei der primären Knieendoprothetik hat der primäre Patellarückflächenersatz keinen klinischen Vorteil gezeigt [10].

\section{Chirurgische Technik: intraartikuläre Resektion und tumorendoprothetische Rekonstruktion (zementfrei)}

Nach korrekter Lagerung des Patienten in Rückenlage, optional mit Fußkeilkissen und Seitstütze sowie Gabe einer SingleShot-Antibiose, erfolgt der Hautschnitt über eine lange mediale oder laterale Inzision von der Mitte des Oberschenkels über parapatellar medial laufend bis zur Tuberositas tibiae. Die längs verlaufende Biopsienarbe wird spindelförmig umschnitten und verbleibt am Präparat. Der mediale Zugang gewährleistet eine gute Exposition des femoralen GefäßNerven-Bündels, der Fossa poplitea, des Streckapparats und des distalen Femurdrittels inkl. Kniegelenk. Nach Mobilisierung der Vasa femoralis superficialis wird das Intervall zwischen M. rectus femoris und M. vastus medialis dargestellt. Anschließend wird der M. vastus intermedius exploriert. Das Ausmaß der Weichteiltumorausdehnung entscheidet, inwieweit Anteile der Quadrizepsoder Adduktorenmuskulatur reseziert und am Präparat verbleiben müssen. Der M. rectus femoris ist funktionell zur Stabilisierung des Kniegelenks entscheidend. Nach Darstellung der Muskulatur wird das Kniegelenk in $90^{\circ}$ Flexion aufgestellt und im Falle einer fehlenden Invasion des Tumors in das Kniegelenk die Kniegelenkkapsel eröffnet und nachfolgend diese am tibialen Ansatz komplett durchtrennt. Nach Durchtrennung der
Kreuzbänder und Resektion der Menisken werden anschließend die verbliebenen Muskelgruppen vom dorsalen Femur (Gastrocnemiusköpfe) abgelöst. Entsprechend des präoperativ ausgemessenen Sicherheitsabstands wird nun die Femurosteotomie durchgeführt. Nach Abgabe des Resektats zur histologischen Untersuchung werden nach Abgabe der Schnellschnittpräparate aus den Schnitträndern (Femurmarkraum und ggf. Weichteile) sowohl die Handschuhe als auch das Instrumentarium gewechselt.

Muss eine extraartikuläre Resektion durchgeführt werden, wird entlang der Kniegelenkkapsel präpariert und diese mitreseziert. Hier ist die Gefäßdarstellung in der Fossa poplitea aufgrund der anatomischen Nähe einmal mehr wichtig. Die Quadrizepssehne wird patellarseitig präpariert und die Patella in der Koronarebene osteotomiert.

Nach Abgabe des Präparats erfolgt die Rekonstruktion. Zunächst beginnend mit der tibialen Osteotomie (ca. $1 \mathrm{~cm}$ subchondral) mit anschließender Präparation des tibialen Markraums für die tibiale Schaftkomponente mit Implantation des individuellen Prothesentyps. Nun wird analog der femorale Markraum unter schrittweiser Aufbohrung für den jeweiligen Prothesentyp vorbereitet. Nach Implantation der femoralen Schaftkomponente wird das tibiale PE-Plateau eingesetzt und abschließend die Prothese konnektiert. Als Orientierungspunkt für die Rotationseinstellung dient femoral die Linea aspera. Schon während des Aufbohrens und Raspelns des femoralen Markraums sollte dies beachtet werden. Die Beinlänge sollte anatomisch rekonstruiert werden. In jedem Fall ist eine zu starke Traktion auf die neurovaskulären Strukturen zu vermeiden (cave: N. peroneus). Modulare Prothesentypen erlauben intraoperativ eine jederzeit mögliche Feinmodulation für Rotation und Länge unter Einbeziehung der Höhe des PE-Inlays. Anschließend erfolgt die Rekonstruktion der Weichteile, wobei insbesondere die Rekonstruktion des Streckapparats entscheidend für die Funktionalität ist. Sowohl Hamstrings als auch M. sartorius können unterstützend genutzt werden. In Abhängigkeit vom Defekt ist der Transfer des medialen oder lateralen Anteils des M. gastrocnemius möglich. Die Verwendung eines Anbindungsschlauchs wird am distalen Femur nicht genutzt. 


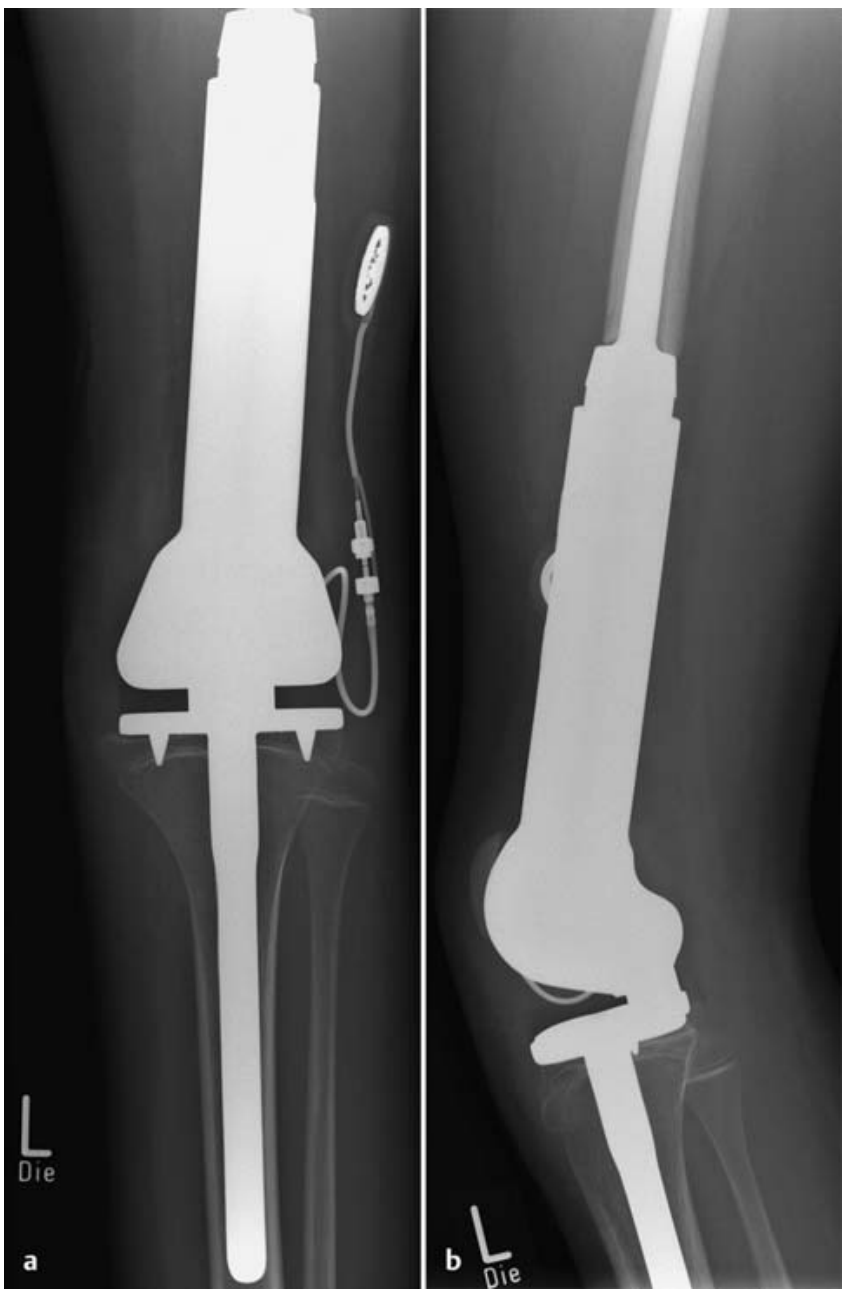

Wachstumsprothesen

Eine Sonderform der Rekonstruktion nach distaler Femurresektion im Wachstumsalter sind sog. „Wachstumsprothesen“ (Abb. 3) mit nicht invasivem Verlängerungsmechanismus. Aufgrund des kontinuierlichen Skelettwachstums und daraus resultierender Beinlängendifferenz im weiteren Verlauf sind Tumorresektionen im Wachstumsalter mit deutlichen funktionellen Einschränkungen verbunden. In der Vergangenheit waren daher mehrere Prothesenwechsel notwendig, um diese Unterschiede auszugleichen, was zu einem unzureichenden Beinlängenausgleich mit deutlich erhöhtem Komplikationsrisiko bei multiplen Revisionen führte. Die distale Femurepiphyse trägt im Wachstumalter $\mathrm{zu}$ ca. $1 \mathrm{~cm}$ Längenwachstum pro Jahr bei [1]. In der Vergangenheit wurden teleskopisch verlängerte Prothesen und Kallusdistraktionen angewendet [12]. Im Allgemeinen besteht die Indikation für Wachstumsprothesen bei Kindern mit offenen Wachstumsfugen und einer zu
Abb. 3 a und $\mathbf{b}$ Implantation einer Wachstumsprothese nach Resektion eines Osteosarkoms des distalen Femurs bei einer 9-jährigen $\mathrm{Pa}$ tientin mit integriertem elektromotorisch betriebenem Expansionsmechanismus und subkutan implantiertem Sensor zur perkutanen Fernsteuerung der Expansion.
Komplikationen, wie septische oder aseptische Lockerungen, bei insgesamt akzeptablem funktionellem Ergebnis deutlich höher als bei nicht extendierbaren Endoprothesen [12]. Bei geringgradigen Beinlängendifferenzen können bei noch offener Wachstumsfuge auf der kontralateralen Seite definitive oder temporäre Epiphyseodesen angeboten werden.

\section{Komplikationen}

Tumorendoprothesen sind mit einer zügigen Mobilisation, raschen Vollbelastbarkeit, guten Funktion und kurzen Rehabilitation assoziiert.

Allerdings zeigen sog. Megaprothesen u. a. aufgrund ihrer Größe, dem Rekonstruktionsausmaß mit entsprechendem Weichteilverlust, der relativ langen Operationszeit, ausgedehnten Wundflächen und nach (neo)adjuvanten Therapiemaßnahmen mit Immunsuppression infolge der (Radio-)Chemotherapie eine relativ hohe Komplikationsrate (2592\%) [16]. Diese ist verglichen mit der oberen Extremität aufgrund der größeren Belastung an der unteren Extremität deutlich gesteigert. Neben dem Lokalrezidiv ist die periprothetische Infektion die schwerwiegendste Komplikation. Weitere häufig vorkommende Risiken sind die aseptische Lockerung, der Prothesenverschleiß im Kniegelenk und die periprothetische Fraktur. Grimer et al. führten eine retrospektive Langzeitanalyse von Tumorendoprothesen mit einem minimalen Follow-up von 25 Jahren durch [16]. Von 564 Patienten hatten nach Therapie eines primär malignen Knochentumors 230 überlebt. 102 dieser Patienten wurde ein distaler Femurersatz implantiert. 18\% der Patienten benötigten keine weitere Operation. Im Durchschnitt sind im Verlauf 2,7 weitere Operationen pro Patient zu erwarten. Während das Risiko für ein Lokalrezidiv mit der Zeit abnimmt, persistiert dieses für eine Infektion mit $1 \%$ pro Jahr.

\section{Periprothetische Infektion}

Beim distalen Femurersatz liegt die Inzidenz der periprothetischen Infektion (Abb. 4) bei ca. 11\% [14,29]. Infolge Zementierungen mit Antibiotikazusatz und Modifikation der Implantatoberfläche (Silberbeschichtung) konnte die Infektionsrate reduziert werden [22]. Dennoch verbleibt ein konstantes Lebensrisiko von $1 \%$ pro Jahr [16]. Am häufigsten manifestiert sich eine Infektion in den 
ersten 2 postoperativen Jahren [16]. Die möglichen Therapieverfahren bei einer periprothetischen Infektion bei distalem Femurersatz sind die Lavage mit Polyethylenwechsel bei einem Frühinfekt, der einzeitige Prothesenwechsel, der zweizeitige Wechsel und die sekundäre Amputation. Ein implantaterhaltendes Vorgehen ist nur bei einem sicheren Früh- oder akuten Spätinfekt sinnvoll. Bei einem Spätinfekt ist zunächst die Explantation und zweizeitige Replantation bei klinisch und paraklinisch sicher beherrschter Infektsituation zu bevorzugen. Intraoperativ wird eine jeweils ausreichende Probengewinnung $(\geq 5)$ empfohlen [23], um eine resistenzgerechte Antibiose auch nach Replantation zu ermöglichen. Am häufigsten werden koagulasenegative Staphylokokken (48\%) und polymikrobielle Keime (26\%) nachgewiesen [29]. In der Langzeitstudie von Grimer et al. konnten über ein zweizeitiges Vorgehen $70 \%$ der tiefen Infekte beherrscht werden. Andernfalls droht die Amputation (30\%). Bei der Explantation ist auf ein knochensparendes Entfernen der häufig festsitzenden Prothese zu achten. Gegebenenfalls müssen zur Replantation Sonderprothesen, größere Megaprothesen oder gar ein totaler Femurersatz implantiert werden. In der Primärendoprothetik wird eine Infekteradikation bei bekannter Resistenzlage durch einen einzeitigen Wechsel diskutiert [31]. Diesbezüglich ist die Erfolgsrate bei Megaprothesen in wenigen Studien noch sehr unsicher (42-78\%) [27, 29]. Bei fehlender Beherrschung der Infektion ist die sekundäre Amputation zu erwägen. Insbesondere Patienten mit adjuvanter Strahlentherapie neigen diesbezüglich zu einem erhöhten Risiko (55,5\%) [21].

\section{Mechanische Komplikationen}

Aseptische Schaftlockerungen sind aufgrund der Belastung häufig (7-11\%) [14, $25,33,35]$. In der Regel ist nach Ausschluss eines Infekts der einzeitige Prothesenwechsel möglich. Gegebenenfalls muss eine geringe Nachresektion im Bereich des Schafttellers infolge des Verlusts an Knochensubstanz erfolgen. Bei einem zementfreien Wechsel ist zur Sicherung einer Osteointegration auf einen längeren Schaft mit größerem Durchmesser zu achten [23]. Bei älteren Patienten oder fehlender Knochensubstanz wird die Zementierung empfohlen [23].

Brüche des Implantats (Abb. 5) waren in der Vergangenheit häufig (3,3-15\%),

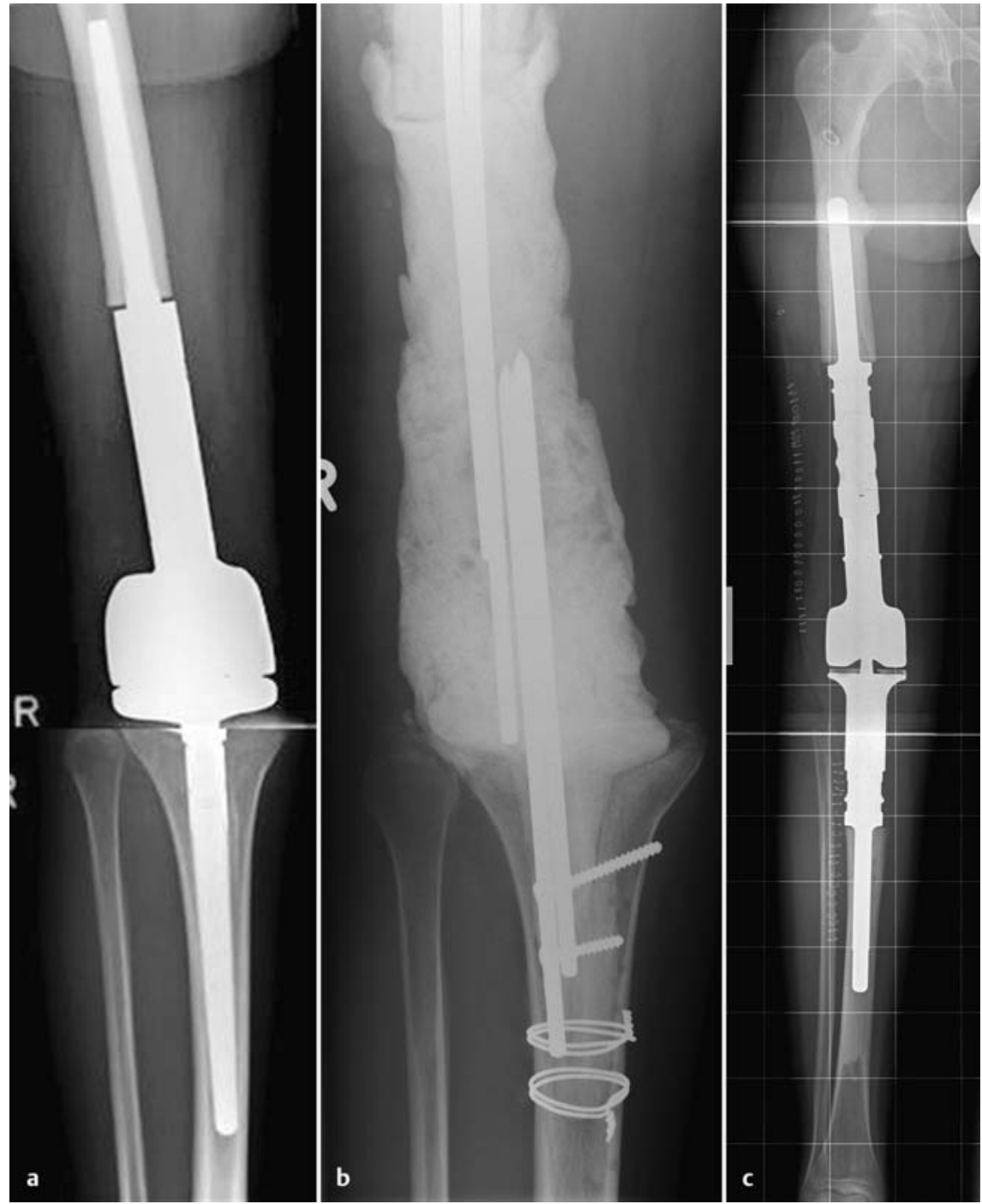

Abb. 4a bis d a 20-jähriger Patient: distaler Femurersatz nach Resektion eines Osteosarkoms. b 15 Monate postoperativ Revision mit radikalem Débridement, Explantation des Femurersatzes und Spacer-Implantation bei Infektion. c, d Replantation nach gesicherter Infekteradikation mit zusätzlich notwendiger Rekonstruktion der proximalen Tibia.

sind allerdings heutzutage selten geworden $[14,15,33,35]$. Gegebenenfalls müssen insbesondere bei zementfreien Verankerungen transfemorale Osteotomien zur Bergung des Implantats durchgeführt werden.

Nach Einführung von Rotating-HingeGelenken ist die Verschleißrate des Kopplungsmechanismus (Abb.6) deutlich geringer geworden. Insgesamt sollte bei klinischen Beschwerden und nachgewiesenem Gelenkverschleiß ein zeitnaher Wechsel erfolgen, um eine sekundäre aseptische Schaftlockerung zu vermeiden. Jede Wechseloperation ist allerdings mit einem signifikanten Risikofaktor für eine periprothetische Infektion verbunden [29].

\section{Periprothetische Fraktur}

Im Allgemeinen sind bei Megaprothesen keine größeren Studien oder eine vergleichbare Klassifikation wie bei der Primärendoprothetik bekannt. In Abhängigkeit von der Frakturlokalisation, Stabilität der Schaftverankerung und Knochenqualität erfolgt die weitere Therapie. Nicht dislozierte Frakturen mit stabilem Implantat können ggf. konservativ therapiert werden. Alternativ können Draht-Cerclagen zur Anwendung kommen. Eine weitere Alternative stellen winkelstabile Plattenosteosynthesen, idealerweise minimalinvasiv-perkutan eingeschoben, dar. Voraussetzung ist das fest integrierte Implantat und die Möglichkeit einer ausreichenden Schrauben- 


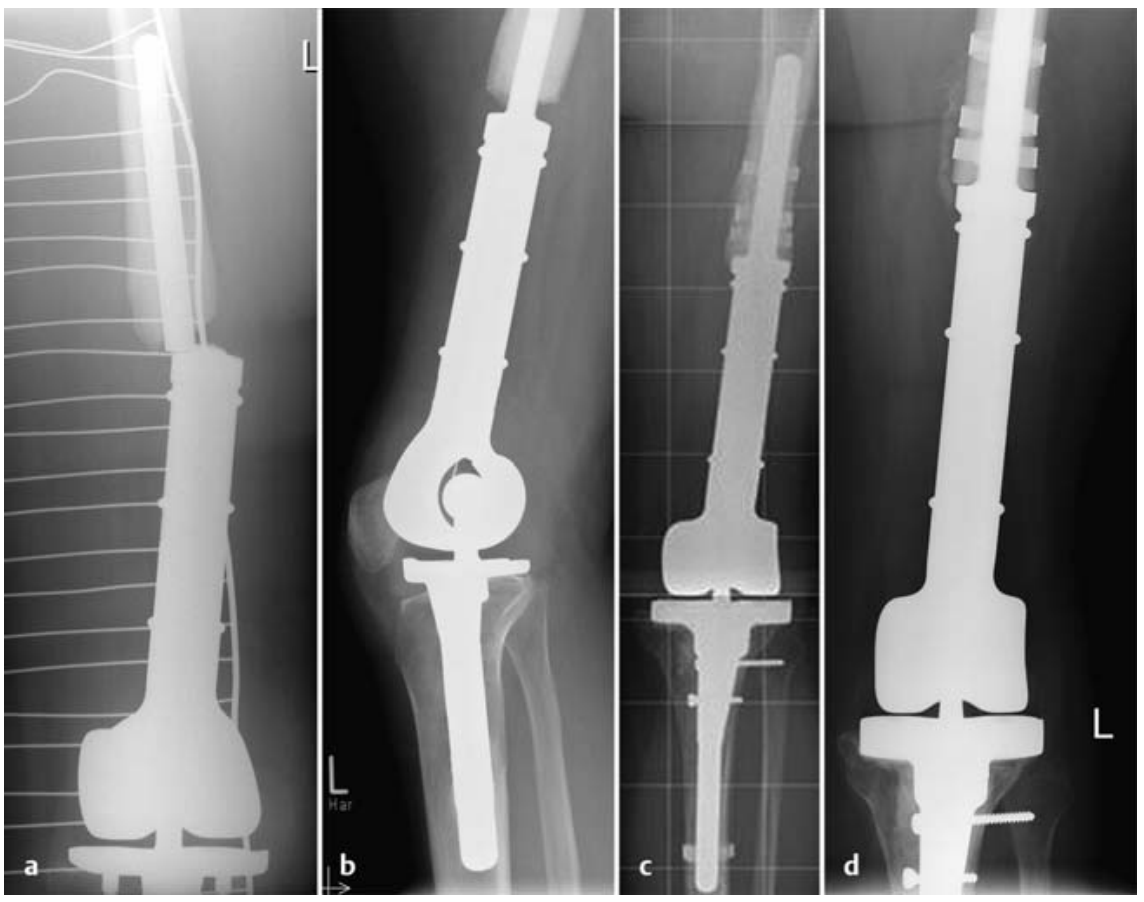

Abb. $\mathbf{5}$ a bis $\mathbf{d}$ a, b 29-jährige Patientin: Implantatversagen 7 Jahre nach distalem Femurersatz bei Osteosarkom. c, d Kompletter Wechsel mit transfemoralem Zugang, Sicherung durch DrahtCerclagen und Implantation eines distalen Femurersatzes mit modifiziertem Kopplungsmechanismus.
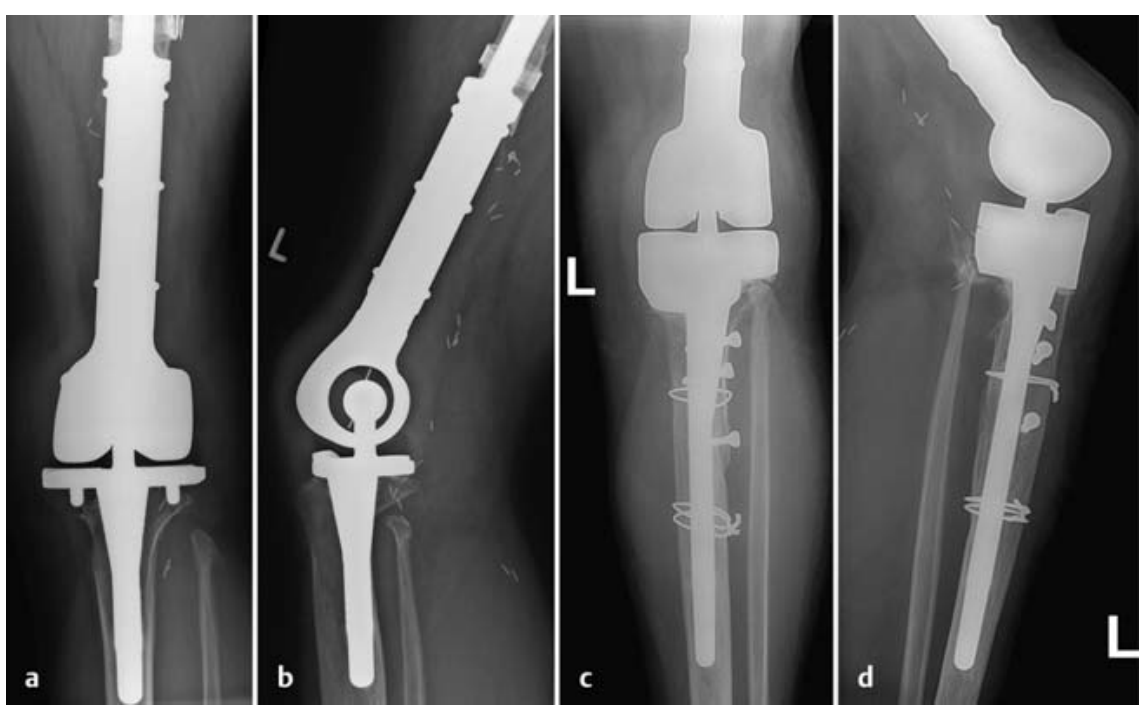

Abb. 6a bis d 28-jähriger Patient 7 Jahre nach distalem Femurersatz bei Osteosarkom mit Schmerzen und Instabilitätsgefühl bei Verschleiß des Kopplungsmechanismus (a, b), kompletter Wechsel mit modifiziertem Kopplungsmechanismus und intraoperativer Notwendigkeit transtibialer Osteotomie bei fest verankerter Tibiakomponente (c, d).

fixierung. Bei Lockerung der Prothese kann durch einen Wechsel auf einen längeren Schaft und die additive Nutzung von Draht-Cerclagen die Fraktur überbrückt und stabilisiert werden. Bei mangelnder Knochenqualität ist ggf. eine zementierte Rekonstruktion notwendig.

Interessenkonflikt: Nein.

\section{Literatur}

${ }^{1}$ Aguilar JA, Paley J, Santpure $S$ et al. Clinical validation of the multiplier method for predicting limb length at maturity, part I. J Pediatr Orthop 2005; 25: 186-191

2 Bacci G, Ferrari S, Longhi A et al. Non-metastatic osteosarcoma of the extremity with pathological fracture: Local and systemic control by amputation or limb salvage after preoperative chemotherapy. Acta Orthop Scand 2003; 74: 449-454

${ }^{3}$ Bielack S, Jürgens $H$, Jundt $G$ et al. Osteosarcoma: the COSS experience. Cancer Treat Res 2009; 152: 289-308

4 Bus MP, van de Sande MA, Fiocco M et al. What are the long-term results of MUTARS ${ }^{\circledR}$ modular endoprostheses for reconstruction of tumor resection of the distal femur and proximal tibia? Clin Orthop Relat Res 2015; [Epub ahead of print]

${ }^{5}$ Capanna R, Morris HG, Campanacci D et al. Modular uncemented prosthetic reconstruction after resection of tumours of the distal femur. J Bone Joint Surg Br 1994; 76: 178186

${ }^{6}$ Chandrasekar CR, Grimer RJ, Carter SR et al. Modular endoprosthetic replacement for tumours of the proximal femur. J Bone Joint Surg Br 2009; 91: 108-112

Cole AS, Hill GA, Theologis TN et al. Femoral nailing for metastatic disease of the femur: a comparison of reamed and unreamed femoral nailing. Injury 2000; 31: 25-31

8 Donati F, Di Giacomo G, Ziranu A et al. Silver coated prosthesis in oncological limb salvage surgery reduce the infection rate. J Biol Regul Homeost Agents 2015; 29 (4 Suppl.): S149S155

${ }^{9}$ Enneking WF, Spanier SS, Goodman MA. Current concepts review. The surgical staging of musculoskeletal sarcoma. J Bone Joint Surg Am 1980; 62: 1027-1030

10 Etchebehere M, Lin PP. Bird JE et al. Patellar resurfacing does it affect outcomes of distal femoral replacement after distal femoral resection? J Bone Joint Surg Am 2016; 98 : 544-551

${ }^{11}$ Freyschmidt J, Ostertag H, Jundt G. Knochentumoren: Klinik, Radiologie, Pathologie. Berlin, Heidelberg, New York: Springer; 1998

12 Futani $H$, Minamizaki T, Nishimoto $Y$ et al. Long-term follow-up after limb salvage in skeletally immature children with a primary malignant tumor of the distal end of the femur. J Bone Joint Surg Am 2006; 88: 595-603

${ }^{13}$ Gebert C, Hardes J, Hoffmann C et al. Chirurgische Therapieoptionen bei malignen Knochentumoren. Chirurg 2002; 73: 1162-1169

${ }^{14}$ Gosheger G, Gebert C, Ahrens $H$ et al. Endoprosthetic reconstruction in 250 patients with sarcoma. Clin Orthop Relat Res 2006; 450: 164-171

15 Grimer RJ, Carter SR, Tillmann RM et al. Endoprosthetic replacement of the proximal tibia. J Bone Joint Surg Br 1999; 81: 488-494

16 Grimer RJ, Aydin BK, Wafa $\mathrm{H}$ et al. Very longterm outcomes after endoprosthetic replacement for malignant tumours of bone. Bone Joint J 2016: 98-B: 857-864

17 Guder W, Hardes J, Gosheger G et al. Osteound Chondrosarkome des Beckens und der unteren Extremitäten. Chirurg 2015; 86: 993-1003

18 Guzik G. Results of the treatment of bone metastases with modular prosthetic replacement - analysis of 67 patients. J Orthop Surg Res 2016; 11: 20

${ }^{19}$ Hage WD, Aboulafia AJ, Aboulafia DM. Incidence, location, and diagnostic evaluation of metastatic bone disease. Orthop Clin North Am 2000; 31: 515-528

${ }^{20}$ Hardes J, Gebert C, Hillmann A et al. [Rotationplasty in the surgical treatment plan of primary malignant bone tumors. Possibilities and limits]. Orthopade 2003; 32: 965-970

${ }^{21}$ Hardes J, Gebert C, Schwappach A et al. Characteristics and outcome of infections associated with tumor endoprostheses. Arch Orthop Trauma Surg 2006; 126: 289-296 
${ }^{22}$ Hardes J, von Eiff C, Streitbuerger A et al. Reduction of periprosthetic infection with silver-coated megaprostheses in patients with bone sarcoma. J Surg Oncol 2010; 101: 389395

23 Hardes J, Ahrens H, Gosheger G et al. Komplikationsmanagement bei Megaprothesen. Unfallchirurg 2014; 117: 607-613

24 Heisel C, Kinkel S, Bernd L et al. Megaprostheses for the treatment of malignant bone tumours of the lower limbs. Int Orthop 2006; 30: 452-457

25 Henderson ER, Groundland JS, Pala E et al. Failure mode classification for tumor endoprostheses: retrospective review of five institutions and a literature review. J Bone Joint Surg Am 2011; 93: 418-429

26 Hillmann A, Hoffmann C, Gosheger G et al. Malignant tumor of the distal part of the femur or the proximal part of the tibia: endoprosthetic replacement or rotationplasty. Functional outcome and quality-of-life measurements. J Bone Joint Surg Am 1999; 81: 462 468

27 Holzer G, Windhager $R$, Kotz R. One-stage revision surgery for infected megaprostheses. J Bone Joint Surg Br 1997; 79: 31-35

28 Höll S, Schlomberg A, Gosheger G et al. Distal femur and proximal tibia replacement with megaprosthesis in revision knee arthroplasty: a limb-saving procedure. Knee Surg Sports Traumatol Arthrosc 2012; 20: 2513-2518

${ }^{29}$ Jeys LM, Grimer RJ, Carter SR et al. Periprosthetic infection in patients treated for an orthopaedic oncological condition. J Bone Joint Surg Am 2005; 87: 842-849
${ }^{30}$ Kawai A, Lin PP, Boland PJ et al. Relationship between magnitude of resection, complication, and prosthetic survival after prosthetic knee reconstructions for distal femoral tumors. J Surg Oncol 1999; 70: 109-115

${ }^{31}$ Leonard $H A$, Liddle AD, Burke O. Single- or two-stage revision for infected total hip arthroplasty? A Systematic review of the literature. Clin Orthop Relat Res 2014; 472: 10361042

32 Mankin HJ, Lange TA, Spanier SS. The hazards of biopsy in patients with malignant primary bone and soft-tissue tumors. J Bone Joint Surg Am 2006; 64: 1121-1127

33 Mittermayer F, Krepler P, Dominkus $M$ et al. Long-term followup of uncemented tumor endoprostheses for the lower extremity. Clin Orthop Relat Res 2001; 388: 167-177

${ }^{34}$ Myers GJ, Abudu AT, Carter SR et al. Endoprosthetic replacement of the distal femur for bone tumours: long-term results. J Bone Joint Surg Br 2007; 89: 521-526

35 Plotz W, Rechl H, Burgkart $R$ et al. Limb salvage with tumor endoprostheses for malignant tumors of the knee. Clin Orthop Relat Res 2002; 405: 207-215

36 Pohlig F, Kirchhoff C, Lenze $U$ et al. Percutaneous core needle biopsy versus open biopsy in diagnostics of bone and soft tissue sarcoma: a retrospective study. Eur J Med Res 2012; 17: 29

37 Schaser KD, Bail HJ, Haas NP et al. Treatment concepts of benign bone tumors and tumorlike bone lesions. Chirurg 2002; 73: 11811190
38 Schmolders J, Koob S, Schepers P et al. [The role of a modular universal tumour and revision system (MUTARS ${ }^{\circledR}$ ) in lower limb endoprosthetic revision surgery - outcome analysis of 25 patients]. Z Orthop Unfall 2016; DOI: 10.1055/s-0042-114704

39 Streitburger A, Gosheger G, Dieckmann R et al. Bone defect reconstruction in bone sarcoma surgery: tumour endoprosthesis versus biological reconstruction. Unfallchirurg 2014; 117: 600-606

40 Winkelmann W. Orthopädie und orthopädische Chirurgie. Tumoren, tumorähnliche Erkrankungen. Stuttgart, New York: Thieme; 2005

Dr. med. Hagen Fritzsche

Wissenschaftlicher Mitarbeiter

PD Dr. med. Christine Hofbauer

Sektionsleitung Tumororthopädie

Prof. Dr. med. Klaus-Dieter Schaser

Ärztlicher Direktor

Zentrum für Orthopädie und

Unfallchirurgie

Universitätsklinik Carl Gustav Carus

Dresden

Fetscherstraße 74

01307 Dresden

hagen.fritzsche@uniklinikumdresden.de 